\title{
Ferdinand Hodler kombiniert im Bild „Enttäuschte Seele“ Alter und Enttäuschung mit einer „Facies leontina“
}

\author{
Ferdinand Hodler Combined in his Picture „Enttäuschte Seele“ an Old Aged Man with Facies Leontina
}

Autor

E. G. Jung

\section{Bibliografie}

DOI 10.1055/s-2007-966079

Akt Dermatol 2007; 33:

92-95 ๑ Georg Thieme

Verlag KG Stuttgart · New York ISSN 0340-2541

Korrespondenzadresse

Prof. Dr. Ernst G. Jung

Maulbeerweg 20 .

69120 Heidelberg

Ernst.G.Jung@t-online.de

\section{Zusammenfassung \\ $\nabla$}

In seinem 1889 gemalten Bild „Enttäuschte Seele“ stellt Ferdinand Hodler einen alten, melancholischen und geknickten Mann dar, der re-

Das Alter beschäftigt uns Menschen seit jeher. Es gab offenbar schon in den frühen Dokumenten menschlicher Lebensgemeinschaften immer Menschen, deren Lebensalter 50 oder 60 Jahre überschritt. Etwa $10 \%$ werden es gewesen sein. Und diese Relation blieb konstant von den alten Kulturnationen bis ins 18. Jahrhundert, also bis vor 250 Jahren. Im selben Zeitraum verbleibt auch die Charakterisierung des Alters und damit dessen Darstellung weitgehend dieselbe [1]. Über die ganze Zeitspanne heben sich zwei divergente, sehr charakteristische Muster ab:

Alter als Fluch: das negative, durch Regression gekennzeichnete Muster des Alters mit Verlust von Fähigkeiten, Abbau von Leistungen, sozialem Abstieg, Isolierung, Schmerzen, Leiden und Vorbereitung zum Sterben. Dargestellt wird der Alte entstellt, mit groben Gesichtszügen, Haarverlust und Verkrüppelung. Er wird nicht ernst genommen, lächerlich gemacht, verspottet und oft geplagt und gequält. Diese Vorstellung geht auf Aristoteles (384-322 v. Chr.) zurück.

Alter als Segen: Dem steht das positive Muster diametral gegenüber: der Greis als weises, würdiges Oberhaupt der Sippe, als Respektsperson, gefragter Ratgeber, Senator, Doge, Fürst, als Wahrer von Überlieferung und Tradition. Dargestellt wird er als stattlicher Mensch mit edlen Zügen, wallendem Haar, traditioneller Kleidung und Stock. Diese Vorstellung geht auf Cicero (106-43 v. Chr.) zurück.

Seit dem 18. Jahrhundert nimmt die allgemeine Lebenserwartung der Menschen in den Kulturnationen infolge hygienischer, medizinischer und sozialer Maßnahmen stetig zu und zudem signierend auf seinen Weggang wartet. Zusätzlich ist eine zentrofaziale Facies leontina dargestellt, wahrscheinlich Ausdruck eines kutanen Lymphoms.

steigt auch der Anteil an Alten (Senioren, nach Maßgabe des Rentenalters) bis gegen $50 \%$. Der negative oder der positive Aspekt bleiben bestimmend, hinzu kommt aber eine Differenzierung der Muster und eine Durchdringung des Alters durch Alterskrankheiten, die jedes Organsystem, oder, multimorbid, alle Organe zusammen betreffen können. Im Vordergrund stehen die Stützorgane (Verkrüppelung), Stoffwechselkrankheiten (Gicht, Diabetes), Herz und Kreislauf, sowie das Nervensystem (Lähmungen und Demenz). Sowohl treffliche wie auch bizarr verzerrte Darstellungen von Menschengruppen oder besonderen Einzelschicksalen erscheinen in der Literatur, und die Aspekte derselben werden in der darstellenden Kunst abgebildet. Während die Literatur Alter und Krankheiten sehr subtil und differenziert zu Biographie und Wirkungsverlauf ordnen und gruppieren vermag, muss die darstellende Kunst Abfolge und chronologisches Zusammenwirken auf einer Abbildung vereinen. Kontamination und Abstraktion mögen hierzu behilflich sein. Zunehmend kommen aber auch vielfältige Gestaltungsentwürfe des Alters [2] zur Sprache und zur Darstellung.

\section{Dermatologie in der Kunst}

$\nabla$

Eine Kulturgeschichte des Alters wird geschrieben [1]. Dabei werden körperliche Beeinträchtigungen dargestellt und solche von besonderen Empfindungen wie Schmerz, Verzweiflung und Unvermögen. Aber auch „Zustände der Seele“ werden trefflich ins Bild gebracht, wie Hoff- 


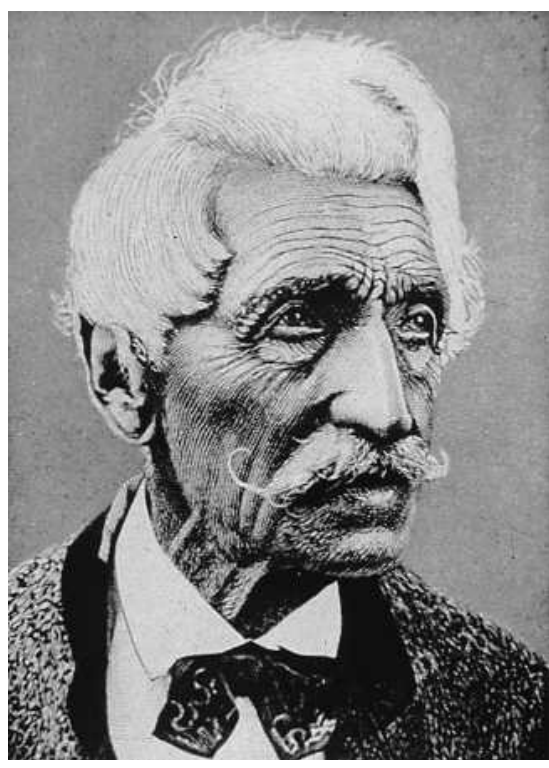
feinen und groben, stehenden Falten im Gesicht; chronologische Alterung verstärkt durch Lichtalterung beim Pionier der Naturheilkunde und Sonnenenthusiasten Arnold Rikli (1823-1906).
Abb. 1 Alte Haut mit

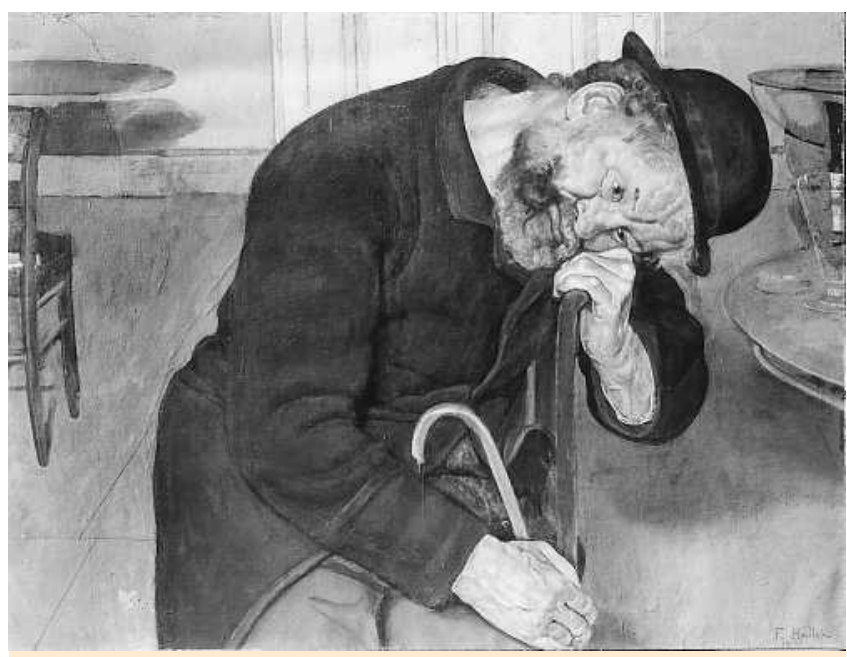

Abb.2 Ferdinand Hodler, Enttäuschte Seele, Öl auf Leinwand, 1889, s-w-Photo @ Kunstmuseum Basel, Martin Bühler.

die Krankheit als Hauptgegenstand wirkt, gleichsam zur Aufklärung und Abschreckung. Solche Werke stellen eine besondere Form der präventiven Information dar und dienten als Warnung zur Verhinderung von Infektionen und von Geschlechtskrankheiten. Karikaturen vermögen solche Aussagen wohl noch zu fokussieren und bedeutend zu steigern.

Der Medizinhistoriker und der Dermatologe sind natürlich versucht, aus solchen Bildberichten die Hautveränderungen und Erkrankungen zu deuten und mit dem heutigen diagnostischen Inventar zu beurteilen. Dies mag in besonderen Fällen schlüssig oder plausibel erscheinen, kann aber nicht darüber hinweg täuschen, dass solche „Spätdiagnosen“ allein aufgrund einer Abbildung, mit oder ohne Beschreibung, äußerst problematisch sind und immer mit Vorbehalten behaftet bleiben $[3,4,7]$.

Betrachten wir die bildhafte Darstellung von Alter und diejenige von Hautkrankheiten, so fällt auf, dass diese beiden Intensionen oft vermischt werden. Dies ist verständlich, da eine ganze Reihe von Hautkrankheiten erst im vorgerückten Lebensalter gehäuft auftreten, diese also alterskorreliert sind. Dennoch fällt auf, dass immer wieder Hautkrankheiten und die Darstellung von Gebrechen beigezogen werden, um die Aussage „Alter als Fluch“ zu untermauern, drastisch zu verstärken. Hautkrankheiten dienen dann nicht nur zur Aussage, dass diese eben im Alter gehäuft auftreten und also noch dazu kommen, sondern sie werden, fast unspezifisch, als Verstärkungselement dem Alter zugeschrieben. Darauf will ich anhand eines Bildes den Blick lenken. Fällen, keineswegs aber ausschließlich, um die Darstellung von Veränderungen und Krankheiten der Haut und deren Anhangsgebilden handelt. Dermatologische Kostproben durchziehen also die Malerei und die Welt der Skulpturen in allen Epochen und Stilrichtungen $[3,4]$. Dabei lassen sich unterschiedliche Intensionen auseinander halten.

Zum einen bildet der beflissene Porträtist die Eigenheiten, die Veränderungen und die Hautkrankheiten seiner Objekte beobachtungsgetreu ab, sodass diese dem Betrachter ins Auge stechen oder, da sie eigentlich Nebensächlichkeiten darstellen, erst nach beflissenem Suchen auffallen.

Zum zweiten gibt es Darstellungen von Hautkrankheiten, die als wichtiger Teil der Abbildung oder gar als deren Hauptaussage imponieren. Damit wird ein Porträt zum Krankheitsbericht gewandelt, der Träger also entsprechend stigmatisiert. Es kann auch sein, dass der Träger sogar in den Hintergrund tritt und

\section{Ferdinand Hodler: Enttäuschte Seele, 1889 $\nabla$}

Dieses Bild, Öl auf Leinwand, $71,5 \times 93,5$, hängt im Kunstmuseum Basel ( $\bigcirc$ Abb. 2) im großen Umgang. Ferdinand Hodler (1853 - 1918) hat das Bild im Alter von 36 Jahren in einer Phase des künstlerischen und religiösen Umbruchs seines Wirkens gemalt. Er vollzog den stilistischen Durchbruch von der flächenhaften symbolischen Ausdrucksmalerei zur Ideenmalerei. Das herausragende Bild dazu ist die „Nacht“, welches 1889/90 entstand und mehrfach preisgekrönt wurde. Es hängt jetzt im Kunstmuseum Bern. Von acht jungen Menschen liegen die meisten schlafend im Bild, angedeutet parallel, nur zwei Körper sind teilweise erhoben. Symbolisch ist Schlaf, Tod und auch Sexuali- 


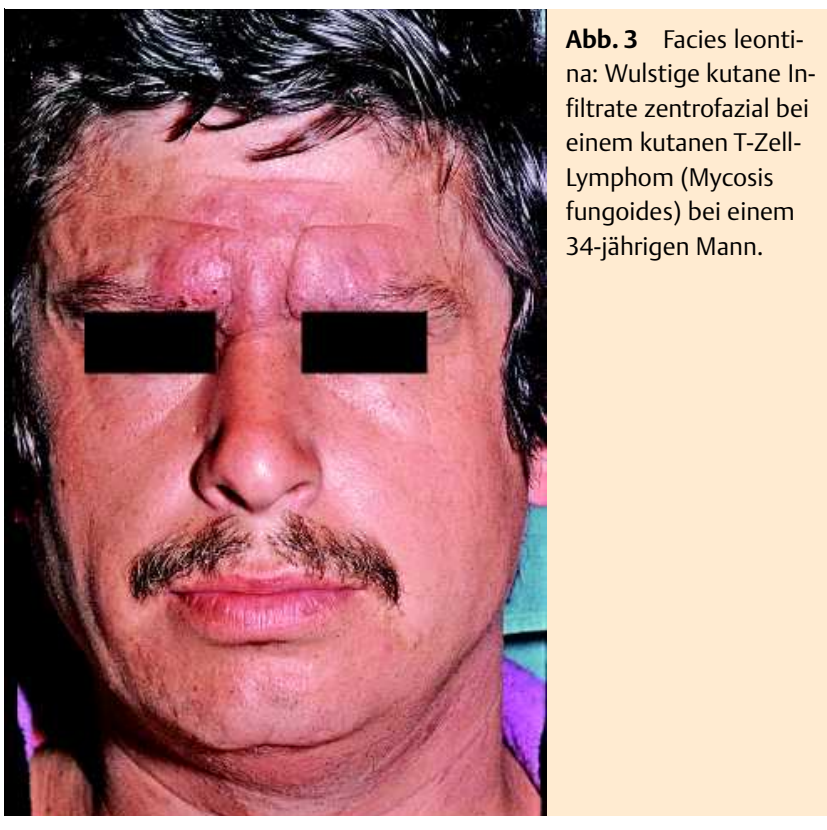

tät ausgedrückt. Der Tag-Nacht-Rhythmus wird im Bild „Der Tag“ 1900 mit fünf parallel dargestellten jungen Männern und Frauen seine Fortsetzung finden. Diese erheben sich mit tanzender Geste aus dem Sitzen lebenslustig in den morgendlichen Tag (Kunstmuseum Bern).

Eine andere Gedankenfolge führt vom Bild „Nacht“ zum 1892 entstandenen Gemälde „Die Lebensmüden“ (Neue Pinakothek München), in welchem fünf bärtige Männer in sitzender Stellung im typischen Parallelismus dargestellt werden. Sie warten alt und eingefallen, die Hände betend gefaltet auf ihr Ende. Bis zum Jahre 1895 beschäftigte sich Ferdinand Hodler in Dutzenden von Zeichnungen und Bildern mit der Darstellung des Alters, wobei die Verknüpfung mit Armut und Melancholie vorherrscht [5].

Dazwischen ist das 1889 entstandene Bild „Enttäuschte Seele“ anzusiedeln. Es stellt eine Einzelfigur dar, einen alten Mann, geknickt auf einem Stuhl sitzend, stützt er den Kopf auf die Stuhllehne. Das Gesicht ist im rechten Halbprofil gezeigt. Der Ausdruck ist müde und hoffnungslos, eben enttäuscht, und die offenen Augen sind gleichsam nach innen gerichtet. Mit Rock und Hut gekleidet und dem Stock als Altersattribut wartet er gelassen auf den Weggang. Die „enttäuschte Seele“ ist als alter, resignierender Mann mit all diesen Attributen dargestellt. Eine gewisse Ähnlichkeit des Kopfes kann man zur Figur links außen im Bild „Die Lebensmüden“ erkennen, obschon dieser frontal dargestellt ist. Passen könnte es. Hodler hat diesen Gedankenzug nach 1895 nicht mehr aufgegriffen, vielmehr hat er sich dem Symbolismus einerseits mit heroisierenden Parallelbildern und der Landschaftsmalerei zugewandt.

Betrachten wir nun im Bild „Enttäuschte Seele“ die Haut etwas genauer, so kommt noch ein weiteres Moment mit ins Spiel. In der Gesichtsmitte, sichtbar an Stirne, Nase und Wangen (Mund und Kinn sind bartverhangen) finden sich unscharf begrenzte, knollige Infiltrate mit leichter Rötung. Die Haut erscheint wulstig aufgetrieben mit groben Knoten und Furchen. Ähnliche Veränderungen finden sich auch am linken Vorderarm und ganz diskret am rechten Handrücken. Die Haut ist also nicht nur auffällig „aufgedunsen“ [5], sondern krankhaft strukturiert. Dies sind keine Altersveränderungen, sondern distinkte Zeichen einer systemischen knotigen Hauterkrankung. Kratzspuren als Zei-

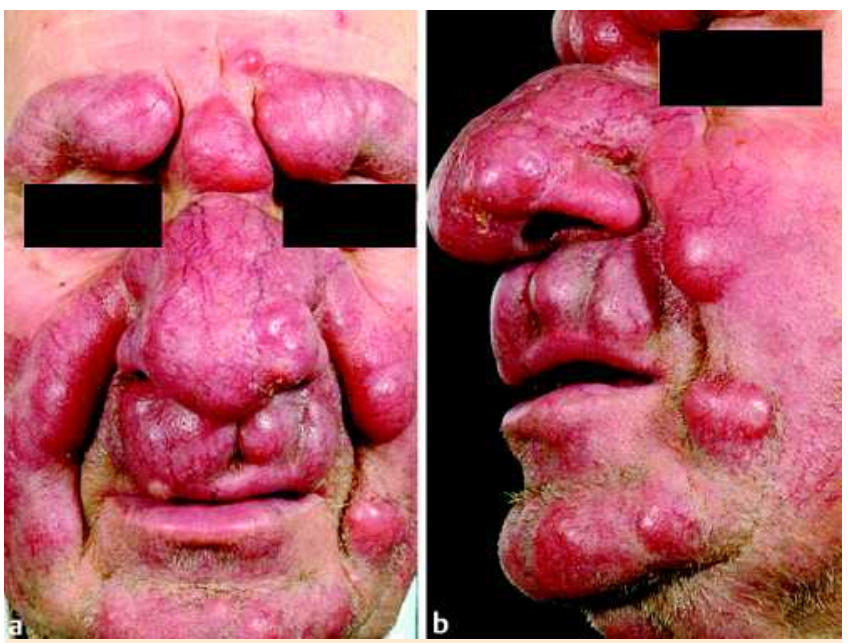

Abb. 4 Facies leontina: Kutanes zentrofaziales Infiltrat bei chronischlymphatischer Leukämie [7]. (Akt Derma 2005; 31:389, Abb. 1)

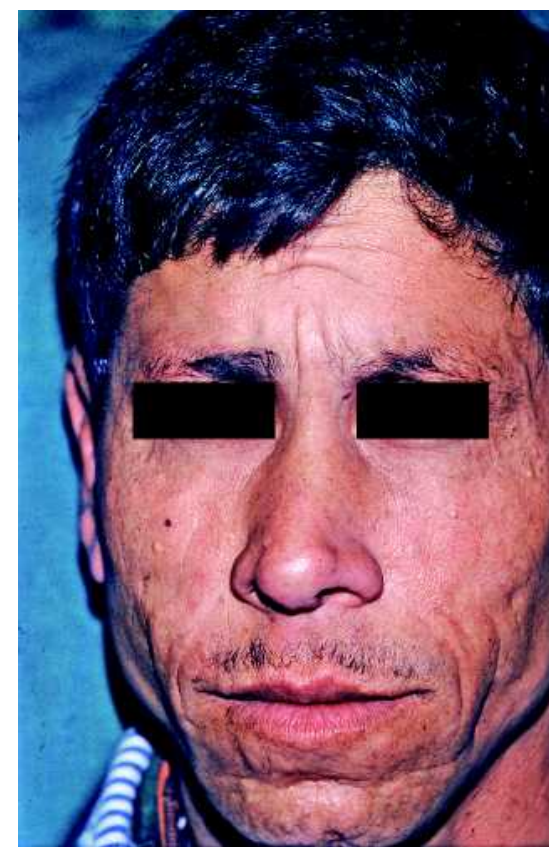

Abb. 5 Zentrofaziale kutane Infiltrate einer lepromatösen Lepra bei einem 39-jährigen Mann.

chen von Juckreiz finden sich nicht. Ferdinand Hodler hat also dem Alten ganz bewusst und gezielt seine Hautkrankheit mitgemalt. Möglicherweise hat er diese als betontes Zeichen der Hautalterung aufgefasst oder aber als Alters-assoziierte Hautkrankheit. Möglicherweise war letzteres im Vordergrund und hat als progrediente Systemerkrankung an der Haut zur Enttäuschung des Alten beigetragen. Da wir über die dargestellte Person keine Informationen haben, kann eine Krankengeschichte seiner Haut nicht rekonstruiert werden. Bei aller Vorsicht retrograder Diagnostik aufgrund von Abbildungen darf hier doch von einer Facies leontina (Löwengesicht) gesprochen werden. Ja, Hodlers Bild zeigt eine solche in geradezu typischer, ja klassischer Lokalisation und Ausprägung ( $\bullet$ Abb. 2). Der teilweise Verlust der Augenbrauen könnte passen. Die Facies leontina stellt eine deskriptive Diagnose dar, welche aufgrund verschiedener Krankheiten, pathogenetischer Entitäten, zustande kommt. Am häufigsten steckt ein kutanes Lymphom dahinter, eine bösartige Systemerkrankung mit Wucherung krankhafter Blutzellen (Lymphozyten), die sich zuerst und besonders in und unter der Haut ansam- 
meln, große, weiche und wenig schmerzarme Knoten bilden, großflächig die Haut besetzen und zuletzt, früher oder später, zur Invasion in den gesamten Organismus und zum Tode führen. Diese Diagnose hat große Wahrscheinlichkeit, müsste aber durch Histologie und Zytologie ergänzt werden. Dies ist jedoch im vorliegenden Fall nicht mehr möglich. Zum Vergleich sind zwei Fälle von Facies leontina bei Mycosis fungoides ( $\odot$ Abb. 3) und bei chronisch-lymphatischer Leukämie ( 0 Abb. 4) beigefügt [6]. Dequeker und Kollegen [8] vermuten, dass schon im 16. Jahrhundert eine Mycosis fungoides zur Darstellung kam, obschon deren Charakter damals noch nicht bekannt war. Kutane Lymphome kommen in allen Lebensaltern vor, sind also keine Alters-assoziierte Erkrankungen, wenn man von der chronischlymphatischen Leukämie absieht.

Auch infektiöse Erkrankungen können in seltenen Fällen zum Bild einer Facies leontina führen, wie Lepra ( $\bullet$ Abb. 5) oder Tuberkulose. Während die Lepra im ausgehenden 19. Jahrhundert in der Schweiz weder endemisch noch in regionalen Kleinepidemien auftrat, also unwahrscheinlich erscheint, kam die Tuberkulose damals in allen Formen und in erschreckender Häufigkeit vor. Ferdinand Hodler kannte die Tuberkulosekrankheit, verlor er doch mehrere Familienmitglieder an Lungen-Tuberkulose. Eine Haut-Tuberkulose kann also nicht ganz ausgeschlossen werden, obschon diese „fressende Flechte“ (Lulus vulgaris) einen anderen Aspekt hat mit Mutilationen und Vernarbungen, was im Bild ausdrücklich nicht dargestellt ist.

\section{Abstract}

\section{Ferdinand Hodler Combined in his Picture \\ „Enttäuschte Seele“ an Old Aged Man with \\ Facies Leontina \\ $\nabla$}

Ferdinand Hodler painted in „Enttäuschte Seele“ an old, melancholic and broken man, who resigned and waits for going away. The picture shows in addition a centrofacial Facies leontina, may be a manifestation of a cutaneous lymphoma.

\section{Literatur:}

1 Thane P (Hrsg). Das Alter. Eine Kulturgeschichte. Darmstadt: Primus, 2005

2 Baltes PB. Hoffnung mit Trauerflor. Lebenslänge contra Lebensqualität - Von der Menschenwürde im Alter. Zürich: NZZ Nr. 258, 6.11.2006

3 Wagner G, Müller WJ. Dermatologie in der Kunst. Biberach an der Riss: Basotherm $\mathrm{GmbH}, 1970$

4 Reitz $M$. Kunst und ärztliche Diagnose. Expedition in die Wissenschaft, Band 1. Weinheim: Wiley VCH, 2006: 149-169

5 Kaenel P. Fin de vie, fin de siècle. Ferdinand Hodler et la vieillesse. Géronotogie et Société 1998; 87: 94-111

6 Plaza T, Seiler B, von den Driesch P. Rosacea-artige kutane Infiltration des Gesichtes (Facies leontina) bei chronisch-lymphatischer Leukämie. Akt Dermatol 2005; 31: 388 - 390

7 Dequeker J. Der Künstler und der Arzt. Ein anderer Blick auf Gemälde. Leuven: Davidsfonds NV, 2006

8 Dequeker J, Degreef H, Busschots A-M, Mallia C. Mycosis fungoides in a painting by Lambert Lombard (1506-1566). Dermatology 2002; 205: $78-79$ 